\title{
An Overview of Belgian Legislation Applicable to Biobank Research and Its Interplay with Data Protection Rules
}

\author{
Teodora Lalova, Anastassia Negrouk, Laurent Dollé, Sofie Bekaert, \\ Annelies Debucquoy, Jean-Jacques Derèze, Peggy Valcke, Els J. Kindt, \\ and Isabelle Huys
}

\footnotetext{
T. Lalova $(\square)$

Department of Pharmaceutical and Pharmacological Sciences, KU Leuven, Leuven, Belgium

Centre for IT and IP Law (CiTiP), KU Leuven, Leuven, Belgium

e-mail: teodora.lalova@kuleuven.be
}

\begin{abstract}
A. Negrouk
European Organisation for Research and Treatment of Cancer, Brussels, Belgium

e-mail: anastassia.negrouk@eortc.org

L. Dollé

Biothèque Wallonie Bruxelles (BWB), Department of Pathology, Erasme Hospital,

Brussels, Belgium

\section{S. Bekaert}

Department of Public Health and Primary Health Care, Faculty of Medicine and Health

Sciences, Ghent University, Ghent, Belgium

e-mail: sofie.bekaert@ugent.be
\end{abstract}

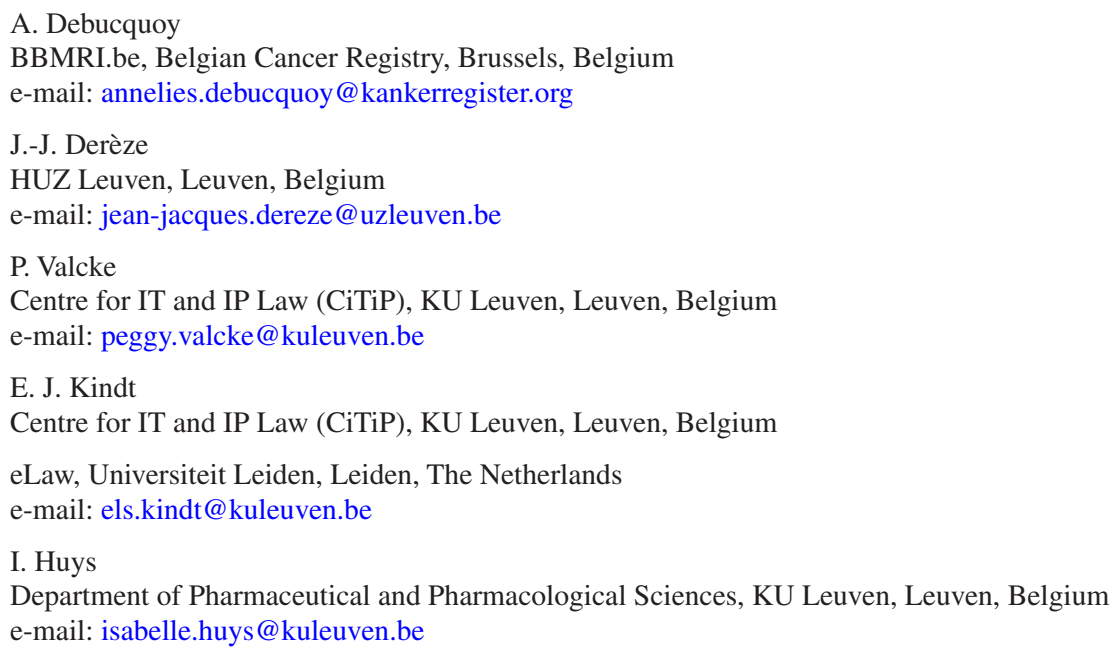




\begin{abstract}
This contribution aims to present in a clear and concise manner the intricate legal framework for biobank research in Belgium. In Part 1, we describe the Belgian biobank infrastructure, with a focus on the concept of biobank. In Part 2, we provide an overview of the applicable legal framework, namely the Act of 19 December 2008 on Human Body Material (HBM), and its amendments. Attention is given to an essential piece of self-regulation, namely the Compendium on biobanks issued by the Federal Agency on Medicine Products and Health (FAMPH). Furthermore, we delineate the interplay with relevant data protection rules. Part 3 is dedicated to the main research oversight bodies in the field of biobanking. In Part 4, we provides several examples of the 'law in context'. In particular, we discuss issues pertaining to presumed consent, processing of personal data associated with HBM, and information provided to the donor of HBM. Finally, Part 5 and 6 addresses the impact of the EU General Data Protection Regulation (GDPR), suggests lines for further research, and outline the future possibilities for biobanking in Belgium.
\end{abstract}

\title{
1 Biobank Infrastructure
}

\subsection{What Is a Biobank}

The applicable Belgian legislation defines a biobank as 'the structure which, for the purpose of scientific research, with the exclusion of research with human medical applications, obtains, processes, stores and makes available human body material, and, where appropriate, the associated data relating to the human body material and the donor' (see Figure 1). ${ }^{1}$ It is sufficient to carry out one of the activities listed above for scientific purposes in order to be characterized as a biobank. ${ }^{2}$ Such structure may be established within an accredited hospital or faculty of medicine and health sciences, or it may also be created outside of a hospital, for instance by a private organization, such as a pharmaceutical company. ${ }^{3}$ The law further requires the positive opinion of an ethics committee concerning the objectives and activities of the biobank. ${ }^{4}$

Human body material (HBM) is defined as 'any human biological material, including human tissues and cells, gametes, embryos, foetuses, as well as substances derived therefrom, and regardless of the degree of their transformation, with the exception of substances of non-human origin'. ${ }^{5}$

\footnotetext{
${ }^{1}$ Article 2 (27) of the Act of 19 December 2008 regarding the procurement and use of human bodily material destined for human medical applications or for scientific research applies, hereafter the Act on HBM.

${ }^{2}$ For the sake of completeness, it must be noted that the Belgian Act on HBM defines three additional structures that collect HBM and associated data. These are the bank for HBM, the intermediate structure of HBM and the production establishment, all for therapeutic purposes, see Article 2 (24) (25) and (26). The rules for biobanks do not apply to these structures, and vice versa.
}

${ }^{3}$ Sterckx and Van Assche (2011), p. 249.

${ }^{4}$ Article 22(1) (3) of the Act on HBM.

${ }^{5}$ Article 2 (1) of the Act on HBM. 
The majority of Belgian biobanks are organized at a central level, within the framework of an institution, e.g. a hospital or a university. In such situation, these hospitals/universities require their researchers to use that central biobank infrastructure. However, it is possible that even a sole researcher can be regarded as a biobank and will have to abide to the strict applicable legislation. This will be the case if his activity formally falls within the scope of the Belgian biobank legislation. ${ }^{6}$

The biobank manager (often referred to in literature as custodian) is the central responsible authority in the Belgian biobank infrastructure. ${ }^{7}$ The custodian must be a doctor who fulfils the conditions laid down in Article 25 of the Law on the exercise of health care professions or a national of one of the Member States of the European Union who is authorized to practice medicine in a Member State other than Belgium. ${ }^{8}$ The rights and responsibilities of the custodian, as elaborated in the Act on HBM, are critical for the conduct of biobanking activities and translational biomedical research. ${ }^{9}$

\subsection{Types of Biobanks and Biobank Networks in Belgium}

On a broader European level, there are many different types of biobanks, established for various purposes and reasons. A report issued by the European Commission (EC) has provided a classification of biobanks that can be translated to the Belgian

\footnotetext{
${ }^{6}$ Examples are provided in the Compendium, issued by the Federal Agency for Medicines and Health Products (FAMHP) in order to address the most pertinent questions in regards the biobank legislation. For instance, if HBM is stored by a researcher for future research and he has not concluded an agreement with a biobank for the storage of the samples, the research himself would be under an obligation to notify himself as a biobank. See the FAMHP Compendium (2018), p. 4 and 9 .

${ }^{7}$ However, the legislation features another figure that is entrusted with responsibility vis-à-vis specific biobank activities. This is the so called 'operator' ('exploitant' or 'uitbater' in the French, respectively Dutch language versions of the Act on HBM). Firstly, a biobank that deals with gametes, embryos or foetuses shall be exclusively operated by the operator of an approved laboratory on research on in-vitro embryos, see Article 3(4)(9) of the Act on HBM. Secondly, and more generally, it is required from the operator to conclude the agreement with the third persons or institutions to which HBM is made available, as established in Article 22 (2) (3) of the Act on HBM.

${ }^{8}$ Article 2 (28) of the Act on HBM.

${ }^{9}$ Some specific responsibilities are further elaborated in the Royal Decree of 9 January 2018 on biobanks, in implementation of Article 22 of the Act of December 2008 (hereafter the Royal Decree of 9 January 2018), for instance duties to keep a register on the specific subject of the scientific research (using a predefined template) and responsibilities related to the processing of personal data in the biobank. Opinion No 45 of 19 January 2009 of the Belgian Advisory Committee on Bioethics brings attention to some of the most important custodian duties. These include, inter alia, checking whether the conditions required prior to obtaining HBM are satisfied, whether the associated personal data are limited to those required for research, and whether the data is coded or anonymized in the most suitable way. Furthermore, it is the custodian's responsibility to keep a register of the samples available and an overview of the transfer of HBM made. Finally, he or she is the one responsible for the management of the biobank and for ensuring that the biobank abides to all relevant legal rules, professional directives, and international codes of conduct. The custodian responds to supervisory authorities and to the management committee of the biobank in case a breach of the applicable rules occurs.
} 
context. ${ }^{10}$ According to the authors of the report, there are certain biobank characteristics that can be used to distinguish between different types of biobanks. These include size, research design, the types of biological samples collected, the method of sample collection, processing and storage, and the disease/research focus. ${ }^{11}$ Hence, the following types of biobanks can be enumerated: large-scale biobanks, small collection biobanks, population-based biobanks, disease-oriented biobanks, case-control biobanks, tissue banks, biobanks in the context of clinical trials, and other specific biobanking formats, such as Guthrie cards (newborn screening), cord blood, or stem cells. ${ }^{12}$

It is hard to provide an exhaustive overview of the types of biobanks in Belgium, as the Belgian legislator has not pronounced itself on the matter and because no official record exists. ${ }^{13}$ The only exception concerns the distinction between biobanks created in the context of a clinical trial and biobanks in general.

The sampling and operations carried out on HBM in the context of a clinical trial on medicinal products for human use, are excluded from the scope of the Belgian Act on HBM. ${ }^{14}$ The provisions for biobanks created in the framework of a clinical trial are containted in the Act of 10 April 2014. Attention must be paid to situations where HBM and associated data which were initially collected in a clinical trial, are used later for purposes other than the ones defined in the clinical trial protocol. In this case, the collected data and material would fall within the scope of the Act on HBM. ${ }^{15}$

In addition to the foregoing, the concept of a biobank network must be discussed. The term is not defined in the legislation, however a working definition is established in literature, together with a classification of such networks. A biobank network could be described as 'a group of institutions who freely assume the commitment to collaborate in the domain of biobanking and who (often) share the same procedures and quality policies, and who are (or might be) helped by a central hub for coordination in terms of service'. ${ }^{16}$ In Europe, Biobanking and BioMolecular Research infrastructure-European Research Infrastructure Consortium

\footnotetext{
${ }^{10}$ Gottweis et al. (2012).

${ }^{11}$ Gottweis et al. (2012), p. 13.

${ }^{12}$ Gottweis et al. (2012), pp. 15-16.

${ }^{13}$ However, the Belgian Federal Agency for Medicines and Health Products (FAMHP) is currently working on publishing a list of all notified biobanks, which will shed clarity on the matter, as required by Article 22 (1) (8) of the Act on HBM. At the time of preparation of this Chapter, said list is not yet publicly available, though all historical biobanks (meaning the ones who have been conducting biobanking activities prior February 2018) had to be notified before 1 May 2019 to the FAMHP in order to legally pursue their activities.

${ }^{14}$ Article 3 (3) (f) of the Act on HBM. Note that this applies only to clinical trials as defined in the Act of 7 May 2004 or Regulation (EU) 536/2004.

${ }^{15}$ Article 3 (3) (f) of the Act on HBM.

${ }^{16}$ Morente et al. (2011), p. 188.
} 
(BBMRI-ERIC) $)^{17}$ is the largest and most significant example of a biobank network, as it connects biobanks and researchers from 20 countries. ${ }^{18}$ Belgian's BBMRIERIC node has been in operation since 2013, under the name of BBMRI.be. It unites the historically established Belgian biobank networks, ${ }^{19}$ one of which formally no longer exists. ${ }^{20}$ In the period 2013-2019, BBMRI.be has matured into a solid partner network on biobanks in Belgium and has proven to reach out to a broader community beyond the founding partners. From 2019 onwards, BBMRI.be invites all Belgian biobanks with translational research potential, as well as biobank users that are seeking structural research collaborations with the BBMRI.be network to join the BBMRI.be network.

\footnotetext{
${ }^{17}$ See also the definition provided for BBMRI-ERIC as a biobank network, namely 'a distributed research infrastructure of biobanks and biomolecular resources, which provides [for its Member States] expertise and services (...) and facilitates access to collections of paner biobanks and biomolecular resources', as found on http://www.bbmri-eric.eu/faq/.

${ }^{18}$ Most of the countries participating in BBMRI-ERIC have the status of full Member states (e.g., Austria, Belgium, Bulgaria), while several participate as observers (e.g., Turkey, Switzerland, Cyprus). More information about the national nodes and contact points at http://www.bbmri-eric. eu/national-nodes/.

${ }^{19}$ All historical Belgian network initiatives could be perceived to fall within the type of a catalogue network. According to literature, a catalogue network consists of a central database from which researchers can obtain information whether the participating biobanks provide access to specific HBM and associated data, see e.g., Verlinden (2015), p. 11. See also Shickle et al. (2010) for a detailed distinction between different types of biobank networks, namely storage networks, bringand-share storage networks, catalogue networks, partnership networks, contribution networks, expertise networks, and networks in population cohorts.

${ }^{20}$ These are the Belgian Virtual Tumourbank (BVT), Biothèque de la Fédération Wallonie-Bruxelle (BWB), and the Flemish Biobank Network (which is officially no longer in operation). The BVT is coordinated by the Belgian Cancer Registry and within it 11 hospitals (including all major Belgian university hospitals) cooperate. Within this network, a standardized set of oncological data is collected centrally in an online catalogue that can be consulted by researchers in the field of oncology to identify samples of interest for their research, see more at http://virtualtumourbank. kankerregister.org/tumourbank.aspx?url=BVT_home. The Biothèque de la Fédération WallonieBruxelle (BWB) unifies eight biobanks from the territory of the Walloon and Brussels capital regions. BWB is an inter-university collaboration platform, stated by the Université Catholique de Louvain (UCL), Université Libre de Bruxelles (ULB) and the University of Liège (ULg). At the time of preparation of this Chapter, BWB is funded by Innoviris. The BWB network has an online catalogue providing rapid access to high-quality specimens and associated medical/biomolecular data, compliant with international quality standards and regulations. See more about BWB at http://bwb.creatix.be/. Finally, the partners of the former Flemish Biobank Network are united within BBMRI.be as well. The Flemish Biobank Network was organized between the four Flemish University hospitals and five universities, and established five central biobank facilities, a harmonized quality and ethical-legal framework, and a central catalogue.
} 


\section{Regulatory Environment for Biobank Research in Belgium}

\subsection{Legal Framework for Biobanks}

\subsubsection{The Act of 19 December 2008 on Human Body Material}

The Act on HBM applies to the donation, collection, procurement, control, treatment, storage, distribution, and use of HBM and manufactured products derived from HBM, intended for human applications or for scientific research purposes. ${ }^{21}$

Regarding the scope ratione materiae, the rules of the Act on HBM are applicable to any human biological material. ${ }^{22}$ Although exceptions exist, ${ }^{23}$ the scope of application remains extremely broad. For instance, within the scope fall all derived substances irrespective of their degree of transformation. ${ }^{24}$ It follows from the foregoing that the Act on HBM is in principle also applicable to DNA and proteins. ${ }^{25}$ Gametes, embryos, and foetuses, even if to a limited extent, also fall under the scope of the legislation. ${ }^{26}$ The broad scope of the law ratione materiae has been subject to criticism from stakeholders in the field, as it does not provide for an adequate nuancing of the different types of HBM, and thus imposes too strict regulations in all cases. Such conclusion follows when the most recent proposal for amendment of the Act on $\mathrm{HBM}^{27}$ is taken into consideration.

\footnotetext{
${ }^{21}$ This act was designed to implement Directives 2004/23/EC, 2006/17/EC and 2006/86/EC, as stipulated in Article 1.1 therein. The directives, in contrast to the Act on HBM, relate to human tissues and cells intended solely for application on humans and treatment purposes (see e.g., Article 1 of the Directive 2004/23/EC), and not for scientific research use.

${ }^{22}$ Article 2(1) of the Act on HBM.

${ }^{23}$ Pursuant to Article 3(3)(a)-(e) of the Act on HBM, separate legal frameworks are in force as regards organ transplantations; blood; sampling and operations with HBM for autologous use in the context of a single intervention; sampling and operations carried out for the exclusive purpose of diagnosis for the benefit of the person from whom the body material was collected, and finally, hair, nails, urine, mothers milk, tears and sweat. It should be noted, however, that the Act provides for exceptions to the exceptions. Namely, the collection, storage and making available of blood would still fall under the scope of the Act on HBM, when these activities are carried by a biobank, see Article 3(3)(b). The same applies for the use of hair, nails and other regenerative material, when the intended purpose is scientific research, see Article 3(3)(e).
}

${ }^{24}$ ARTICLE 2(1) and 3(2) of the Act on HBM. 'Transformation' is defined in the law as 'any manipulation that substantially modifies the genetic code of the cells that make up the human body material so that the material does not show a link with the donor and can no longer provide meaningful information about the health status of the donor', see Article 2(3)(7) of the Act on HBM, as translated into English in the FAMHP Compendium (2018), p. 16. Transformation can occur only in the case that the donor of the HBM has consented to that. Human body material can be transformed if the donor has agreed to this.

${ }^{25}$ Verlinden (2015), p. 78.

${ }^{26}$ Article 3(4) of the Act on HBM.

${ }^{27}$ Proposal for legislation, deposited to the Belgian House of Representatives on 21 February 2019, publicly available at: http://www.dekamer.be/FLWB/PDF/54/3589/54K3589001.pdf A critical discussion of the proposal is not within the scope of this Chapter, however in order to better eluci- 
Regarding its scope ratione loci, the Belgian Act on HBM applies, first, to HBM removed on Belgian territory, ${ }^{28}$ but also, second, to samples imported from abroad and used in Belgium. ${ }^{29}$

Finally, regarding the scope of the law ratione personae, three conditions must be fulfilled cumulatively to regard any entity as a biobank: (1) the entity must be carrying out one or more of the activities enumerated in the law (obtains, processes, stores and makes available of HBM and/or associated data); (2) the use of HBM must be done for the purposes of scientific research, ${ }^{30}$ and (3) the activities that a structure has to perform in order to be established as a biobank, include the obtaining, processing, storage and making available of HBM for scientific research. ${ }^{31}$

The initial text of the Act on HBM contained legal rules on the procurement and use of HBM by biobanks for research purposes that did not enter into force for 10 years, and in the meantime were amended several times. Changes were introduced, first, by the Act of 19 March 2013 containing diverse provisions concerning health. Second, the Act of 10 April 2014 containing diverse provisions concerning

date the challenges that the current law poses in practice, it is of importance to bring attention to some of the changes sought. In the preamble, it is acknowledged that the current scope of the biobank rules is too strict vis-à-vis the nature of some type of HBM. Hence, it is proposed that the scope of application of the law is limited to certain key provision when it comes to some materials. Key example is the revision of the notion of 'transformed material'. The proposal introduces two new terms, namely 'artificial' and 'extracted' material, which allow for better nuancing of the nature of the HBM, see Article 3 of the Proposal. 'Artificial material' is to be understood as material that is produced outside the human body, with the main focus being on cell lines, where cells from a human donor have been replaced by 'manufactured' cells. 'Extracted' material, on the other hand, is material that has been cell or tissue extract, but no longer consists of cells, e.g. ribosomes, mitochondria, etc. The legal regime envisaged for these two types of material is less strict, as long as the material is not intended for genetic research. In all cases, however, the proposal maintains the obligation for an ethics committee check of the use of the material. Further, the proposal seeks to remedy problems of interpretation of the law. Finally, it creates a legal basis for the digital sharing of data concerning the health of the patient with the patient himself or healthcare providers, see Chapter 6 of the Proposal, that further builds up on the already existing Belgian eHealth platform, and more specifically on the so-called Personal Health Viewer, available to Belgian citizens on https://www.masante.belgique.be/\#/.

${ }^{28}$ Verlinden (2015), p. 79.

${ }^{29}$ The FAMHP Compendium (2018), p. 6. When that is the case, all imported samples must be registered by a Belgian biobank with which a framework agreement or a contract must be concluded.

${ }^{30}$ Article 2(32) defines 'scientific purposes' as 'any use of human body material with a view to development of the knowledge specific to the exercise of the health care profession as referred to in the law concerning the exercise of health care professions, coordinated on 10 May 2015'. The definition requires to treat operations with HBM with caution, as, for instance, from the moment that HBM enters into a biobank, it will no longer be available for direct clinical use, as stipulated in Article 8(2)(1) of the Act on HBM.

${ }^{31}$ Article 2(27) of the Act on HBM. In cases where HBM is temporarily stored in the context of ongoing scientific research, such temporary storage would not require a researcher to notify himself as a biobank, on the conditions that the researcher has concluded an agreement with a biobank and that the research is conducted within a defined time frame or for a specific purpose, see the FAMHP Compendium (2018), p. 4. 
health established specific rules for biobanks created in the framework of a clinical trial. Finally, The Act of 22 June 2016 introduced further modifications to the legal framework. All three Acts were scheduled to enter into force only after the publication of one or more executive Royal Decrees. ${ }^{32}$ With the adoption of the Royal Decree of 9 January 2018, the legal framework described above finally entered into force. ${ }^{33}$

A recent amendment of the Act on HBM that requires attention, is the Act of 30 October 2018. With its entry into force, the scope of Belgian biobank rules ratione materiae was extended to the donation, procurement, control and import of HBM intended for use exclusively in manufactured products, in particular medicinal products, advanced therapy medicinal products (ATMPs) ${ }^{34}$ or medical devices. ${ }^{35}$ Another significant change brought by the new amendment act, is the establishment of a new service within the Federal Agency for Medicines and Health Products (FAMHP), which should provide advice on access to HBM. ${ }^{36}$

\subsubsection{The Royal Decree of 9 January 2018}

The Royal Decree ${ }^{37}$ establishes rules pertaining to, inter alia, the biobank notification procedure; the collection of human material; the approval by and reporting to ethics committees; the organization of a biobank register; the content of the agreement between a biobank and the recipient of the human substances.

\subsubsection{The Compendium on Biobanks, Issued by the Federal Agency for Medicines and Health Products (FAMHP)}

The Compendium is a form of self-regulation ${ }^{38}$ which strives to shed clarity as to how to interpret and implement the complex system of legal requirements. During the preparation of the Compendium, the input of relevant stakeholders was sought,

\footnotetext{
${ }^{32}$ Article 124 of the Act of 19 March 2013, Article 139 of the Act of 10 April 2014, and Article 45 of the Act of 22 June 2016.

${ }^{33}$ Article 15 of the Royal Decree of 9 January 2018.

${ }^{34}$ Belgian legislation refers directly to the definition for advanced therapy medicinal products provided at European level, namely 'any of the following medicinal products for human use: - a gene therapy medicinal product as defined in Part IV of Annex I to Directive 2001/83/EC, — a somatic cell therapy medicinal product as defined in Part IV of Annex I to Directive 2001/83/EC, - a tissue engineered product', see Article 2(1) of Regulation (EC) No 1394/2007 on advanced therapy medicinal products.

${ }^{35}$ Article 3(1) of the Act on HBM as modified by Article 3 of the Act of 30 October 2018.

${ }^{36}$ The service is titled 'Commité d'allocation du matériel corporel humain' $(\mathrm{CAMCH})$ in French and 'Allocatiecomité voor menselijk lichaamsmateriaal' (ACMLM) in Dutch, translated into English as 'Human Body Material Allocation Committee', see Article 21(3)/1 of the Act on HBM, as amended by Article 15 of the Act of 30 October 2018.

${ }^{37}$ The Royal Decree of 9 January 2018 on biobanks in implementation of Article 22 of the Act of 19 on HBM, entered into force on 01.11.2018.

${ }^{38}$ To be taken as meaning that the relevant stakeholders have voluntarily committed to abide by the guidelines as established in the document.
} 
namely representatives of academic and industrial biobanks, ethical committees and juridical experts. By providing answers to 47 consolidated questions, the document covers a broad range of topics such as, inter alia, the scope of the biobank legislation, consent, notification procedure, transformation of HBM, traceability and anonymization, ethics committees.

\subsubsection{Belgian Data Protection Legislation}

Data protection legislation must always be considered when it comes to biobanking activities. The reason for this lies in the fact that access to associated data ${ }^{39}$ is of crucial importance for the proper conduct of most biomedical research. Limited access to such data could result in a lack of reproducibility and risk of misinterpretation of the research results. ${ }^{40}$

\subsection{5 'Associated Data' as Personal Data}

The Belgian Privacy Commission ${ }^{41}$ brought attention to the fact that information about a number of characteristics of the donor must be provided every time an operation is conducted on HBM. ${ }^{42}$ This is in line with Article 2 (27) of the Act on HBM, which states that within the scope of the law is also 'where appropriate, associated data relating to the human body material and the donor'. The Belgian biobank legislation refers to personal data, although it does not provide a definition of the term itself. However, the Belgian Privacy Commission further established that such biological and medical characteristics of the donor (i.e., associated data) have to be regarded as personal data in relation to the health of the donor in the sense of Article 7 of the Act of 8 December 1992 on the protection of privacy. ${ }^{43}$

\footnotetext{
${ }^{39}$ Associated data includes data related to the donor, such as demographic data, e.g. age and gender, or data on previous diseases or family history, and data about the quality characteristics of the HBM, see Verlinden (2015), p. 4. However, it should be born in mind that personal data is not only collected upon procurement of HBM, but it can also be generated when samples are being processed. Pursuant to Recital 35 of the GDPR, 'personal data concerning health should include (...) information derived from the testing or examination of a body pa or bodily substance, including from genetic data and biological samples'. This brings another layer of complexity to the matter of associated data (as the new generated personal data necessarily will always fall within the special categories of data provided for in the GDPR). Hence, the crucial importance of taking into consideration data protection legislation when conducting biobank activities.

${ }^{40}$ Verlinden (2015), p. 4.

${ }^{41}$ Operating as the Belgian Data Protection Authority since 25 May 2018, as reformed by a law of 16 November 2017 and.

${ }^{42}$ Opinion No 10/2009.

${ }^{43}$ Opinion No 10/2009.
} 


\subsubsection{The Act of 30 July 2018}

The Act of 8 December 1992 was replaced by the Act of 30 July $2018 .{ }^{44}$ It can be argued that the authoritative guidance issued in the past by the Belgian Privacy Commission applies to the new legislation as well.

The Act of 30 July 2018 stipulates that the definitions of GDPR apply directly. ${ }^{45}$ Hence, central concepts such as 'personal data', 'controller', 'processor', or, in the context of biobanking, 'data concerning health' and 'genetic data', are to be understood as they are defined in the GDPR. ${ }^{46}$

\subsubsection{Interplay Between the Belgian Data Protection and Biobank Rules}

In addition to the reference to personal data described above, the Belgian biobank legislation provides for an interplay with data protection rules on several other grounds, listed below.

- Data controller and data subject in the context of biobank research: Responsibility for the processing of personal data in the framework of a biobank is allocated to the biobank custodian. ${ }^{47}$ Logically, the data subject is the donor of HBM. ${ }^{48}$

- Record of processing activities: On the one hand, Article 191 of the Act of 30 July 2018 lists the additional elements that the controller should add to the record of processing activities in case data is processed for scientific purposes. Besides this list, the legislation does not provide any further guidance or template for this record. On the other hand, the Act on HBM establishes that a biobank should keep a 'register' with information about the nature, origin and destination of the stored HBM, ${ }^{49}$ and a template for such a register is provided in the Royal Degree of 9 January 2018. The two types of records complement each other, and the lack of a unified format could be perceived as an additional burden for the data controller/biobank custodian.

- Storage period: The maximum storage period for personal data is 50 years after procurement of HBM. ${ }^{50}$

\footnotetext{
${ }^{44}$ Act of 30 July 2018 on the protection of natural persons with regard to the processing of personal data, which implements the EU General Data Protection Regulation (GDPR).

${ }^{45}$ Article 5 of the Act of 30 July.

${ }^{46}$ See Articles 4(1), 4(7), 4(8), 4(13), and 4(15) of the GDPR.

${ }^{47}$ Article 11(3) of the Royal Decree of 9 January 2018.

${ }^{48}$ Verlinden (2015), p. 85.

${ }^{49}$ Article 22 (2) of the Act on HBM.

${ }^{50}$ Article 22 (8) of the Act on HBM. This provision of Belgian law is directly related to the principle of storage limitation, as established in Article 5(e) of the GDPR. However, the Act on HBM does not contradict the principle of storage limitation, as it benefits from the exception provided for personal data processed solely for scientific research.
} 
- Traceability and identification of personal data: ${ }^{51}$ Traceability is a crucial concept in biobanking. It constitutes the ability to locate and identify HBM and associated personal data at any stage of the biobank process, from procurement to distribution for use, or destruction. ${ }^{52}$ Concerning HBM obtained by a living donor, traceability depends on the consent of the donor or of the person who is legally authorized to grant permission for the procurement of HBM..$^{53}$ In the case of residual HBM obtained from a diseased person, the determination of whether the material should be traceable could be made by several people, which the law enumarates in a limited manner, including the custodian of the biobank. ${ }^{54}$ Hence, such HBM is collected based on a presumed (non-explicit) consent procedure. As traceability is necessarily linked to the processing of personal data, the discussed provisions also bear influence on the degree of control that a donor will have regarding his privacy. ${ }^{55}$

- Further processing of personal data: Pursuant to Article 194 of the Act of 30 July 2018, where personal data are not collected from the data subject, the controller should conclude an agreement with the original controller. The Article 195 lists the essential elements of the agreement: the contact details of the original controller and of the controller of the further processing; or, in cases where derogations from certain data subject's rights (i.e., right to access, right to rectification, right to restriction of processing, and right to object) have been adopted, the reasons why the exercise of these rights is likely to make the achievement of the purpose of further processing impossible or seriously hinder it.

These rules are directly related to Article 21(1), Article 22(2)(3) of the Act on HBM, and Article 10 of the Royal Degree of 9 January 2018, pursuant to which each provision of HBM by a biobank, whether the HBM is transferred to another biobank or a third party, should be subject to a written agreement with the person or institution receiving the material. The agreement should govern the possible processing of the donor's personal data by the entity to which the material is made available. ${ }^{56}$ The biobank legislation requires that this type of agreements containt more elements, than what is prescribed in the data protection rules, e.g. the subject of the scientific research for which the HBM is made available; the

\footnotetext{
${ }^{51}$ Article 22 (9) of the Act on HBM establishes that the rules on traceability and identification of the donor (outlined in Article 22(37)) are to be further worked out in a Royal Decree, with the aim of guaranteeing data protection in accordance with the applicable Privacy legislation. The relevant Royal Decree was finally adopted in 2018 , hence at the current moment they are enforceable.

${ }^{52}$ Article 2(23) of the Act on HBM.

${ }^{53}$ Article 22(4)(1) of the Act on HBM.

${ }^{54}$ Article 22(4)(2) of the Act on HBM.

${ }^{55}$ Moreover, regard shall be held of Article 11 of the GDPR, according to which 'if the purposes for which a controller processes personal data no longer require the identification of a data subject by the controller, the controller shall not be obliged to maintain, acquire or process additional information in order to identify the data subject for the sole purpose of complying' with the GDPR.

${ }^{56}$ Article 22(2)(3) of the Act on HBM.
} 
responsibilities for ensuring traceability; a description of the appropriate technical and organization measures to be taken in the case personal data is also communicated; a coded copy of the consent of the donor.

Finally, Article 11 of the Royal Decree of 9 January 2018, expressly forbids the transfer of personal data to third parties, but permits it if it occurs between biobanks.

Questions remain regarding the practical implementation of the provisions discussed above. For instance, a detailed account of the appropriate technical and organizational measures to be taken in cases of personal data transfers lacks in the current Belgian data protection legislation.

\subsection{Procedure for Samples Collection}

\subsubsection{In Theory}

The procedure for samples collection is established in the Act on HBM. Removal of HBM for scientific research is permitted on the condition that it is performed for a specific purpose. ${ }^{57}$ The aim should be specified, precise and relevant for the scientific research.

Again, attention should be paid again to the fact that associated data, and more specifically personal data, are collected alongside samples. Hence, in the context of HBM procurement, data protection rules apply as well. According to the purpose limitation principle established in Article 5(1)(b) of the GDPR, personal data must be processed for 'specified, explicit and legitimate purpose'. The purpose limitation principle is thus in line with the condition established in the Act on HBM as regards the obtaining of samples. However, in contrast to the Act on HBM, the GDPR allows the possibility for a broad consent for research, as long as ethical oversight is provided. Pursuant to Recital 33, 'it is often not possible to fully identify the purpose of personal data processing for scientific research purposes at the time of data collection. Therefore, data subjects should be allowed to give their consent to certain areas of scientific research when in keeping with recognised ethical standards for scientific research.'

- Informed consent: A central place in the samples collection procedure holds the requirement for informed consent ${ }^{58}$ Consent is also one of the possible legal bases for the valid processing of associated personal data. ${ }^{59}$ According to the biobank legislation, informed consent for biobank research shall be given

\footnotetext{
${ }^{57}$ Article 8(1)(1)(1) of the Act on HBM.

${ }^{58}$ Article 10(1) of the Act on HBM stipulates that as a general principle "the removal of human body material from a living person can only be carried out on an adult donor (...) who has previously consented thereto in accordance with the provisions of Article 10(5)'.

${ }^{59}$ As stipulated in Article 6.1(a), read in conjunction with Article 9(2)(a) of the GDPR.
} 
without prejudice to the applicable data protection rules ${ }^{60}$ Pursuant to the Act on HBM, the donor's consent for biobank research must be given in an informed, conscious and free manner, and it must be written, dated and signed. ${ }^{61}$

An interesting parallel between the GDPR and the biobank rules can be made vis-à-vis the right to withdrawal. The Act on HBM provides for a right to withdraw one's consent that can be exercised at any time before the HBM has been subjected to any action after having been obtained. ${ }^{62}$ It is not necessary to motivate the withdrawal. In literature, this right to withdrawal is perceived as rather symbolic, since the donor loses such it as soon as the custodian stores or processes the HBM. ${ }^{63}$ The GDPR also establishes a right to withdraw consent, ${ }^{64}$ which holds relatively more weight than the same right under the Act of HBM. Hence, consent for the processing of associated data can be withdrawn at any time, and if done, this would mean that the custodian must delete all processed data, unless the data can be processed on another legal ground. However, the right of withdrawal under GDPR does not affect the lawfulness of the processing conducted before withdrawal.

- Distinction between primary and secondary use of HBM: Another important consideration related to the Belgian biobank legal framework pertains to the distinction between primary and secondary use of HBM. Primary use is defined as 'any use of human body material to which the donor has explicitly and specifically given consent in the context of the collection', ${ }^{65}$ whereas secondary use is 'any use of human body material other than that to which the donor has given his consent in the context of the collection'. ${ }^{66}$ In Article 20(1) an informed and explicit consent is required for the secondary use of HBM. However, pursuant to the same provision, in cases where it is impossible to seek consent, or where such a request would be exceptionally inappropriate, the positive opinion of an ethics committee would be sufficient to allow the collection of samples.

- Use of residual HBM: A further essential point relates to the use of residual HBM. Residual HBM is defined as the material collected for "the diagnosis or treatment of the donor which, after a sufficient and relevant part has been stored for the establishing, refining or completing the diagnosis or treatment of the donor on the basis of new scientific data, is redundant in relation to these purpose and could therefore be destroyed' ${ }^{67}$ In the case of residual HBM, consent is presumed, unless, prior to any operation with the material, the donor announced his/

\footnotetext{
${ }^{60}$ See Article $10(7)$ of the Act on HBM.

${ }^{61}$ Article $10(5)$ of the Act on HBM.

${ }^{62}$ Article 10(5)(4)of the Act on HBM.

${ }^{63}$ See Verlinden (2015), p. 81 and Panis and Van Gelder (2008).

${ }^{64}$ Article 7(3) of the GDPR.

${ }^{65}$ Article 2(29) of the Act on HBM.

${ }^{66}$ Article 2(30) of the Act on HBM.

${ }^{67}$ Article 2(33) of the Act on HBM.
} 
her refusal. ${ }^{68}$ The refusal must be addressed to the medical specialist referred to in Article 4(1)(1) of the Act on HBM, or to the chief medical officer of the hospital where the sample was taken.

- Who obtains consent: The person responsible for obtaining the consent must be a medical specialist. ${ }^{69}$

- Sample collection from minors and incapacitated persons: The Act on HBM provides for the possibility to obtain HBM from minors and incapacitated persons (as defined in Article 492 of the Belgian Civil Code) only in cases whereby the collection of samples cannot have serious consequences for the donor and the removal involves cells and tissues that regenerate, or in cases where the removal is carried out with an autologous purpose.${ }^{70}$ Informed consent is required, and it should be given by the donor's representative in accordance with the Belgian Act of 22 August 2002 on Patients' Rights.

- Sample collection from diseased persons: The Act on HBM provides that a presumption, established in Article 10-14 of the Act of 13 June 1986 regarding the removal and transplantation of organs, applies for research biobanking as well. ${ }^{71}$ According to the relevant provisions, anyone who has been domiciled in Belgium for 6 months, is presumed to consent to a sample collection after his death, unless he has expressed his opposition ${ }^{72}$ (orally or in writing, with the municipal administration, their general practitioner, or online via MaSante. Belgique.be). This is known as the 'opt-out' system. A new Royal Decree, issued on 9 February 2020, further details the rules on the registration of declarations of will concerning post mortem removal of HBM and organs. The decree enters into force on 1 July, 2020. Of interest is Article 11, which introduces a sensibilization step. Namely, a month before reaching the age of maturity, all persons will receive a letter which would inform them about the opt-out system, and their right to opt-out or explicitly opt-in. The future consequences of this law cannot be predicted. It may be assumed that the new generation will be more aware of the system of opting-out, in comparison to many people today who do not know about it. However, it can also be argued that as long as the general public is not structurally informed about these provisions, this could induce yet another obstacle in accessing HBM for research purposes. They key lies in setting up information campaigns, which are not yet foreseen by the government.

- The no (commercial) advantage rule: The Act on HBM prohibits that any financial or material advantage is offered or received in exchange for the dona-

\footnotetext{
${ }^{68}$ Article 20(2)(1) of the Act on HBM.

${ }^{69}$ Article 10(5)(6) of the Act on HBM.

${ }^{70}$ Article 10(3) of the Act on HBM.

${ }^{71}$ Article 12 of the Act on HBM.

${ }^{72}$ Soon after the adoption of the Act on HBM, the logic of the Belgian legislator in constructing the cited rule was heavily criticized in literature. For example, Sterckx and Van Assche discuss the illegitimacy of extrapolation of presumed consent for uses of HBM for therapeutic purposes to consent for research uses (see Sterckx and Van Assche 2011). However, at the present moment presumed consent has become accepted in practice.
} 
tion of HBM. ${ }^{73}$ The donor can only receive a compensation for the cost or loss of income that is direct results of the donation. ${ }^{74}$

- Who removes the sample: The categories of health professionals that have the right to physically obtain HBM are listed in Article 2 of the Royal Decree. These are medical doctors, dentists, nurses, midwives, pharmacists and licensees or masters in chemical sciences authorized to perform clinical biology analysis, and finally, holders of the professional title 'medical laboratory technologist'. It is possible that the collection of HBM from a living donor takes place outside of a hospital, as long as this occurs in an environment where health, safety and discretion are guaranteed. ${ }^{75}$

\subsubsection{In Practice}

In general, the institutions strictly follow the rules described above. The reliance on presumed consent for the use of residual HBM has not yet become widespread. Because of the need for clarification on the applicable stipulations, explicit consent is often asked also for residual material, as such material in most cases is not anonymized.

Moreover, it could be argued that it anonymization itself is only possible with the donor's consent. The reason lies in the provision of Article 11 of the Act on HBM, according to which, if important information concerning the donor's state of health has been generated during operations conducted on traceable HBM, an obligation is triggered for the biobank to inform the donor about the discovery.

When HBM is procured for secondary purposes, practice shows that in most cases it is impossible to obtain the donor's consent, or it is excetionally inappropriate to seek it.

\section{Biobank Research Oversight}

\subsection{General Remarks}

In Belgium research oversight in the context of biobanking, other than by the Belgian Supervisory Authority for data protection, is provided by three main bodies. These are ethics committees, the Federal Agency for Medicines and Health Products (FAMHP), and Data Protection Officers, as required by the Act of 30 July 2018, in implementation of the GDPR.

\footnotetext{
${ }^{73}$ Article 6 of the Act on HBM.

${ }^{74}$ As confirmed by the FAMHP Compendium (2018), p. 38.

${ }^{75}$ FAMHP Compendium (2018), p. 29.
} 


\subsection{Ethics Committees}

The positive opinion of an ethics committee is required for the establishment of a notified biobank. ${ }^{76}$ Pursuant to Article 22(1)(3) of the Act on HBM, such opinions can only be given by ethics committees with full competence. ${ }^{77}$

Once a positive ethics opinion has been obtained in view of a biobank's general aims and activities, the biobank can also rely upon it as an approval covering particular projects. ${ }^{78}$ Thus the biobank is alleviated from the burden to seek ethical advice for each new procurement of HBM. ${ }^{79}$

In addition, prior to any secondary use of HBM, an ethics committee must provide a favorable opinion. ${ }^{80}$ The ethics committee decides on the relevance of the secondary use and its purpose, the adequacy of the information provided to the donor, and the sufficient specificity and the scope of the donor's consent. ${ }^{81}$

Finally, in cases where it is impossible to seek the donor's consent, or where such a request would be exceptionally inappropriate, the positive opinion of an ethics committee is sufficient to allow the collection of HBM. Whereby such a situation arises, it is also the ethics committee's responsibility to evaluate whether it appears impossible or exceptionally inappropriate to request the donor's consent. ${ }^{82}$

\subsection{The Federal Agency for Medicines and Health Products (FAMHP)}

All biobanks in Belgium have to submit a notification on their activities to the FAMHP (see also Figure 2). ${ }^{83}$

\footnotetext{
${ }^{76}$ Crucial role is in this respect is played by the Belgian Association of Research Ethics Committees (BAREC). Among its objectives is the provision of support to Belgian ethics committees involved in health care. See more at http://barec.be/index.htm.

In addition, note that an exception from the general rule exists for biobanks created in the framework of a clinical trial. In such cases, the ethics approval given as regard the clinical trial as a whole is also considered sufficient for the valid establishment of biobank activities. See Article 22(1)(6) of the Act on HBM.

${ }^{77}$ In accordance with the Act of 7 May 2004 relating to experiments on humans. For a list of all 25 recognized ethics committees in Belgium, see here https://www.famhp.be/sites/default/files/content/lijst_ecs_-_liste_ce_4.pdf.

${ }^{78}$ Article 22(1)(3), (4), and (5) of the Act on HBM.

${ }^{79}$ Note, however, that the usage of HBM by academic or industrial end-users is still subject to approval by a local ethics committee.

${ }^{80}$ Article 21(1) of the Act on HBM.

${ }^{81}$ Article 21(3) of the Act on HBM.

${ }^{82}$ Article 21(3)(3) of the Act on HBM.

${ }^{83}$ Article 22(1) of the Act on HBM and Article 3 of the Royal Decree. An exception to this rule exists for biobanks that are created in the framework of a clinical trial. In such cases, the approval
} 
For a biobank that has been in operation before the entry into force of the Royal Decree (meaning that samples have already been collected before November 2018), the notification procedure had to be finalized before 1 May 2019, following a 6 months grace period.

For all new biobanks, the notification must be done before the start of any samples collection.

\subsection{Data Protection Officer}

Article 37.1 of the GDPR stipulates that in cases where the core activities of a controller or processor consist of processing special categories of data pursuant to Article 9 (i.e., genetic data, biometric data, and data concerning health) on a large scale, a data protection officer shall be designated. Having in mind the sensitive character of biobank activities and of HBM and its associated data, it is to be concluded that most biobanks would have to appoint such Data Protection Officer (hereafter DPO). The DPO can be perceived to have a critical role in the oversight of biobank research.

The DPO must be designated on the basis of his professional qualities, in particular expert knowledge of data protection law and practices. ${ }^{84}$ The DPO's tasks include, inter alia, informing and advising the controller or the processor, and the employees who carry out processing, of their obligations pursuant to the GDPR and to other relevant national provisions; monitoring compliance with the relevant EU and national data protection provisions, as well as the internal policies of the biobank; providing advice as regards data protection impact assessment; cooperating with the data protection supervisory authority.

The Belgian Act of 30 July 2018 also provides for the designation of a DPO, specifically in the cases where personal data are processed for scientific research purposes and the processing may result in high risk. ${ }^{85}$ When personal data is processed for scientific purposes, the controller must anonymize or pseudonymize it after it is collected. In cases of further processing, it is possible to de-pseudonymize the personal data only when necessary for the research purposes and, where applicable, after consulting the DPO. ${ }^{86}$ Furthermore, under Article 204, the DPO must issue opinions on the use of the various pseudonymization and anonymization methods employed. However, the legislator's decision to createthis obligation may be questioned, as at the current moment there are not enough guarantees that DPOs are sufficiently equipped and educated to provide such opinions.

of the clinical trial itself by the FAMHP replaces the requirement to notify the establishment of the biobank, see Article 22(1)(2) of the Act on HBM.

${ }^{84}$ Article $37(5)$ of the GDPR.

${ }^{85}$ Article 190, read in conjunction with Article 32 of the Act of 30 July 2018.

${ }^{86}$ Article $198-200$ of the Act of 30 July 2018. 


\section{Law in Context: Individual Rights and Public Interests}

\subsection{General Remarks}

Several examples could be provided as regards the question how the legal rules outlined above are applied in practice, and more specifically, how the balance between individual rights and the development of science is struck in Belgium.

\subsection{Issues Pertaining to (Presumed) Consent for Obtaining HBM}

As established above, informed consent constitutes the general principle in biobank research for the valid procurement of HBM and associated data. However, consent is absolutely required only in situations whereby samples are collected for primary use.

For secondary use of HBM for research purposes, the Belgian legislation gives the possibility to procure HBM without consent. This will be the case if it is impossible to seek the donor's consent (for instance, the donor is deceased), or if such a request would be exceptionally inappropriate. In such instances, the positive opinion of an ethics committee would be sufficient to allow the collection of samples. ${ }^{87}$

Even more significantly, the concept of presumed consent for residual use of HBM is part of Belgian law. It is always presumed that consent has been given, unless the donor has explicitly refused before any operation was performed on the samples. ${ }^{88}$ This could be seen as a unique 'opt-out' consent system with very practical roots.

The concept of informed and explicit consent has had a central place in biomedical research since it was first embedded in the Nuremberg code.$^{89}$ It is inextricably linked to the principles of human dignity and autonomy, and to the protection of the privacy of the individual, and it is seen as the practical implementation of the right to selfdetermination. ${ }^{90}$ Henceforth, at first glance the Belgian presumed consent system may seem to be in contradiction to the protection of fundamental rights. Indeed, according to some authors the opt-out consent system is 'highly problematic' ${ }^{91}$ However, when discussing the procurement of HBM in practice, and the balance between relevant interests, regard should be held of the following considerations.

The nature of current biomedical research as such calls for the establishment of large pools of samples to ensure genetic representation for the correct testing of

\footnotetext{
${ }^{87}$ Article 21 of the Act of HBM.

${ }^{88}$ Article 20(2)(1) of the Act on HBM.

${ }^{89}$ The Nuremberg Code (1996) 313 BMJ 1448. See also, Kosta (2011) for an overview of the evolution of the concept of consent in the bioethics field, and an elucidation of consent under data protection rules.

${ }^{90}$ Allen and McNamara (2011).

${ }^{91}$ Sterckx and Van Assche (2011), p. 254.
} 
research hypotheses. This is especially prominent as the precision medicine approach is becoming more widespread. If HBM stored by biobanks is unrepresentative of society as a whole, future treatments for those not represented are likely to become increasingly scarce..$^{2} \mathrm{~A}$ practical way to deal with under-representation is presumed consent. When consent has been already obtained for the procurement of HBM for diagnostic/therapeutic uses, going back to the donor for a second consent for research purposes would result in additional costs (as it will require more time and efforts), or it might prove impossible to obtain. Thus, the Belgian presumed consent fosters the development of science. Koslakidis and el. further argue that an opt-out system may be seen as part of an 'altruistic societal obligation' for the common good. ${ }^{93}$ This directly refers to the principle of solidarity, part of the broader bioethical discourse surrounding transplantation for years. For instance, in Belgium, presumed consent for organ donation was established in 1986 with the Law regarding the removal and transplantation of organs. Therefore, the opt-out system in the framework of research biobanking could be viewed as a logical continuation of a long-standing tradition. ${ }^{94}$

\subsection{Issues Pertaining to the Processing of Data Associated to HBM}

With respect to the processing of associated personal data, the GDPR and the implementing Belgian Act of 30 July 2018 apply.

A legal basis is required for the valid processing of personal data. The choice of the correct legal basis is responsibility ofthe data controller. ${ }^{95}$ In practice, there is a lack of sufficient authoritative guidance pertaining to the choice of the most suitable legal basis, and much uncertainty remains.

Recently, the European Data Protection Board (EDPB) issued Opinion No 3/2019 which concerns the interplay between the EU Clinical Trials Regulation (CTR) and the GDPR. At the moment, it is the first EPDB opinion to discuss biomedical research. Biobanking is neither harmonized at EU level, nor regulated in the EU CTR, however, some important conclusions related to it can still be drawn on the basis of Opinion No 3/2019, by way of analogy.

Firstly, the EPDB expressly stipulated that explicit consent ${ }^{96}$ should not always be regarded as the preferred legal basis for the conduct of scientific research. In the

\footnotetext{
${ }^{92}$ Kozlakidis et al. (2012), p. 115.

${ }^{93}$ Kozlakidis et al. (2012), p. 118.

${ }^{94} \mathrm{The}$ adoption of a presumed consent possibility for the valid procurement of HBM offers grounds for a broader critical discussion about the drawbacks and positives of such a system. Such discussion, however, although highly significant from a theoretical point of view, falls outside scope of the present Chapter.

${ }^{95}$ As biobank research deals with sensitive data, the applicable provisions of the GDPR are to be found in Article 9(2) read in conjunction with Article 6.

${ }^{96}$ Article $9(2)(a)$ of the GDPR.
} 
context of a clinical trial, this is especially the case when processing is carried out for reliability and safety purposes, such as e.g., safety reporting or inspection by national competent authorities. The appropriate legal basis, as established by EPDB, is Article 9(2)(i) - 'processing is necessary for reasons of public interest in the area of public health' read in conjunction with Article 6(1)(c)_-'legal obligations to which the controller is subject'.

Secondly, for pure research activities conducted in the framework of a clinical trial, the EPDB rightly acknowledged that the informed consent for participation in a trial must not be confused with consent as legal basis for the processing of data. Extrapolated to the context of biobanking, it seems reasonable to draw a similar distinction as regards the informed consent required to obtain HBM for primary use.

Further, the EPDB brought attention to the imbalance of power between a trial participant and the investigator/sponsor of a trial, which could affect one of the conditions for valid data processing consent, namely that it has to be 'freely given'. In the context of biobank research conducted outside the context of a clinical trial, it could be argued that the power imbalance is not of the same nature, by virtue of the fact that sample donation does not involve the same risks pertaining to possible institutional or hierarchical dependencies that could inappropriately influence a patient's decision to participate in a clinical trial. However, consent should still be regarded with caution when considered as the suitable legal basis for processing. ${ }^{97}$

\subsection{Issues Pertaining to Information Provided to the Donor of HBM}

As established above, pursuant to the Belgian Act on HBM the consent of the donor for the procurement of HBM must be informed. In addition, concerning associated personal data, the GDPR in its Article 13 establishes an information obligation for the data controller. At the time the personal data is obtained, the data subject, i.e. the donor, must receive all of the information specified in Article 13(1) and (2).

The practice in Belgian biobanks is to provide general information about the biobank research via a patient brochure. This general information is not repeated later to the individual donor. It could be argued that, pursuant to Article 13 and 14 of the GDPR, for each research conducted on human body material and for each related processing of personal data, the donor should be individually informed.

\footnotetext{
${ }^{97}$ In the case of use of residual HBM, for instance, the opt-out consent system for the procurement of samples applies in Belgium. Contacting the data subject for consent under GDPR could be seen as an undue burden posed on the biobank custodian. In such instances, another legal basis could be seen as more suitable, e.g. Article 6 (1) (e) of the GDPR, which allows data processing 'necessary for the performance of a task carried out in the public interest' or Article 6(1)(f) - 'for the purposes of the legitimate interests pursued by the controller or by a third pay, except where such interests are overridden by the interests or fundamental rights and freedoms of the data subject'. The logic expressed here follows the one established in the EPDB Opinion No 3/2019. It could be argued that following such reasoning would be beneficial for striking the right balance between individual rights and public interest. However, an in-depth elaboration on this topic is outside the scope of this Chapter, and it provides ground for a separate study.
} 
Furthermore, more specific information is provided by the biobank when the donor's informed consent is obtained. At the current moment a national sensibilization campaign is in development with the aim to inform the general public about the nature and aims of biobank research, similar to organ donation campaigns in the past.

When it comes to information considerations within the Belgian context, one practical challenge might be present in the case of residual use of HBM, as consent for it is presumed, henceforth the donor does not have access to the more specific information that is typically provided during the informed consent procedure. Another unresolved issue is that under the current legal framework, donors cannot indicate that they do not want to receive information. A parallel could be made with the ongoing debate regarding return of research findings in biomedical research and the psychological stress endured by some donors. ${ }^{98}$

\section{GDPR Impact and Future Possibilities for Biobanking}

\subsection{The Impact of GDPR on Biobanking in Belgium}

In addition to the points already presented throughout this Chapter, several more issues related to the impact of GDPR on biobanking in Belgium can be selected for discussion. This part aims to open room for debate and pose questions for further research on the theoretical and practical challenges that the current legal frameworks present.

\subsection{Allocation of Responsibilities According to Biobanking and Data Protection Rules}

Article 5(2) of the GDPR establishes the principle of accountability, according to which the data controller is responsible and should be able to demonstrate compliance with all other data protection principles. In the Belgian biobank framework, the unique concept of custodian is established. According to the law, the custodian can only be a natural person, more specifically a medical doctor, and should fulfill a specific set of strict eligibility conditions..$^{99}$ It is the custodian who is specifically entrusted with the responsibilities of a data controller. ${ }^{100}$ However, the custodian carries a set of additional obligations, assigned to him under the legal framework for biobanking.

It is outside the scope of this article to carry out an in-depth comparative study of the figures of the data controller and the biobank custodian, and more specifically, on how the custodian may exercise most suitably the dual responsibilities allocated

\footnotetext{
${ }^{98}$ De Clercq et al. (2017).

${ }^{99}$ Article 2 (28) of the Act on HBM.

${ }^{100}$ Article 11(3) of the Royal Decree of 9 January 2018.
} 
to him by the two set of applicable rules. However, a meaningful line for further research may concern the influence that the notion of controller has over the performing of custodian duties in practice. Vice versa, insights into how the concept of custodian may bear an impact on the notion of controller in Belgium would be useful. Such research would be of help for the much-needed alignment between data protection and biobank rules.

Further layers of complexity as regards the allocation of responsibilities exists in the field of clinical trials and other interventional studies. As stated in Sect. 1.2 above, the Act on HBM is not applicable to the sampling and operations conducted in the framework of a clinical trial on medicinal products for human use. However, when it comes to studies performed to test medical devices, ${ }^{101}$ studies for in vitro medical devices, ${ }^{102}$ or other types of studies, e.g. a surgical study, no such exception is provided for and the biobank legislation applies in full. A collision might be envisaged between the figures of the sponsor of such a study, the biobank custodian, and the notion of data controller. Pursuant to biobank rules, the custodian is in all cases a data controller. ${ }^{103}$ In the context of an investigational study, it could be argued that the study sponsor would be the data controller, as the natural or legal person that determines the purposes and means of the processing of personal data, associated to the HBM. ${ }^{104} \mathrm{~A}$ more in-depth discussion is necessary as regards the responsibilities of sponsor and custodian in such a context and vis-à-vis the possibilities for joint controllership. Moreover, the foregoing begs further investigation into the national legislator's reasons to exclude only one type of interventional study, namely clinical trials on medicinal products for human use, from the scope of the Act on HBM.

Finally, it is also of interest to discuss the fact that the Act on HBM becomes applicable to data and samples collected in the scope of a clinical trial, if they are later used for other research (i.e., secondary use). To illustrate, we use a hypothetical case, see Fig. 3. First, HBM and associated data are collected and used in the scope of a clinical trial: the Belgian biobank law would not be applicable, and the clinical trial sponsor would be the data controller. The biobank in which, tissues, samples, and associated data are stored, would be sub-contractor of the sponsor, and, moreover, data processor acting on behalf of the sponsor. Second, a number of years after the end of the trial, the sponsor may decide to conduct new research with the previously collected HBM and data. This would be possible, as long as all legal and ethical requirements for secondary use of data are complied with. In this situation, the Belgian biobank law would become available. Regarding

\footnotetext{
${ }^{101}$ Regulated in Belgium by the Royal Decree of 15 July 1997 governing the active implantable medical devices, implementing Directive 90/385/EEC, and by the Royal Decree of 18 March 1999 governing medical devices, implementing and by Directive 93/42/EEC.

${ }^{102}$ Regulated in Belgium by the Royal Decree dated 14 November 2001 governing medical devices for in-vitro diagnostics, implementing Directive 98/79/EEC.

${ }^{103}$ Article 11(3) of the Royal Decree of 9 January 2018.

${ }^{104}$ Article 4(7) of the GDPR.
} 
roles and responsibilities, whereas the sponsor would remain data controller for the original full data set, the biobank manager would turn into joint-controller for the sub data set stored and processed in the biobank. Potential issues emerge. For instance, the biobank remains subcontractor, but pursuant to the Act on HBM, the biobank manager would now have the power to agree or not to the release of samples and data for research. Uncertainties exists also with respect to the agreements for further processing of data and samples, described in Sect. 2.1.7 above, as it is not clear whether the contract between the sponsor and a new recipient of HBM would be sufficient, or whether the biobank would have to sign their own agreement.

\subsection{Allocation of Research Oversight Responsibilities Between Data Protection Officers and Ethics Committees}

Another question that has not been investigated yet and that could present practical challenges in the future, is about the allocation of research oversight responsibilities between DPOs and ethics committees. On the one hand, some of the rights and responsibilities with which DPOs are entrusted seem to require expertise in ethics matters, especially when data processing activities are situated in a biobanking context. For instance, confusion may be brought up by the possibility for a DPO to provide opinion prior to the collection of personal data. ${ }^{105}$ In such cases, it is generally expected that the DPOs would seek the advice of an ethical committee. However, ethics committees themselves are often lacking specific expertise when it comes to data protection matters. The right balance should be sought between these two important actors. Furthermore, efforts in education and cross-sharing of experience are required.

\section{Future Possibilities for Biobanking}

In relation to the many remaining uncertainties in the interpretation of the relevant legal rules, Article 40 of the GDPR offers a welcomed solution by encouraging the drawing up of codes of conducts. The codes of conduct are intended to contribute to the proper application of the data protection legislation in a specific processing sector, and their aim is to overcome fragmentation in implementation. Having in mind the particularly sensitive link between biobanking and data protection, such a comprehensive common interpretation of GDPR norms would have positive influence for fostering biobank research. At present, BBMRI-ERIC is in preparation of a code of conduct. As Belgium is among the few Member

${ }^{105}$ Article 22 of the Act of 30 July 2018. 
States who have a distinct biobank legal framework, it could be envisaged that the Belgian experience in regulating biobanking would be of high significance during the drafting of the code.

A second point to be considered when discussing the future, is collaboration, both on a national level (between biobanks), and on a broader European level. In this respect, the Belgian node of BBMRI-ERIC (BBMRI.be) is currently working on strengthening the harmonization of relevant Belgian policies within the broader framework of BBMRI-ERIC policies. In addition, the structure and governance of BBMRI.be was recently changed to allow biobank users to become part of the network besides the biobank providers. This change is an attempt to improve the interaction and best practices for sharing and mutual understanding of needs and challenges in the use and custodianship of HBM.

\section{Conclusion}

This chapter aimed to shed clarity on the intricate legal framework for biobank research in Belgium, and its interplay with data protection rules. We outlined the key legislative acts and soft law guidance in the field, and critically discussed their practical application. Belgium is among the few countries in Europe which have adopted a specific law for research biobanking. However, gaps and uncertainties remain, especially in relation to the joint application of the biobank and data protection laws. Creating a code of conduct applicable in daily research practice may be the way forward for a pragmatic implementation of all relevant legal and regulatory frameworks. Further suggestions for future investigations and discussions on pertinent questions on the topic were systematically made throughout the chapter.

Acknowledgments TL's PhD is supported by a scholarship awarded by the Research Foundation Flanders (project number: $11 \mathrm{H} 3720 \mathrm{~N}$ ), and is conducted in collaboration with the European Organisation for Research and Treatment of Cancer (EORTC).

\section{Figures}

Figures 1 and 2, have been created by Dr. Laurent Dollé (Biothèque Wallonie Bruxelles), and are used as illustrations on the official website of Biothèque Wallonie Bruxelles as well. 


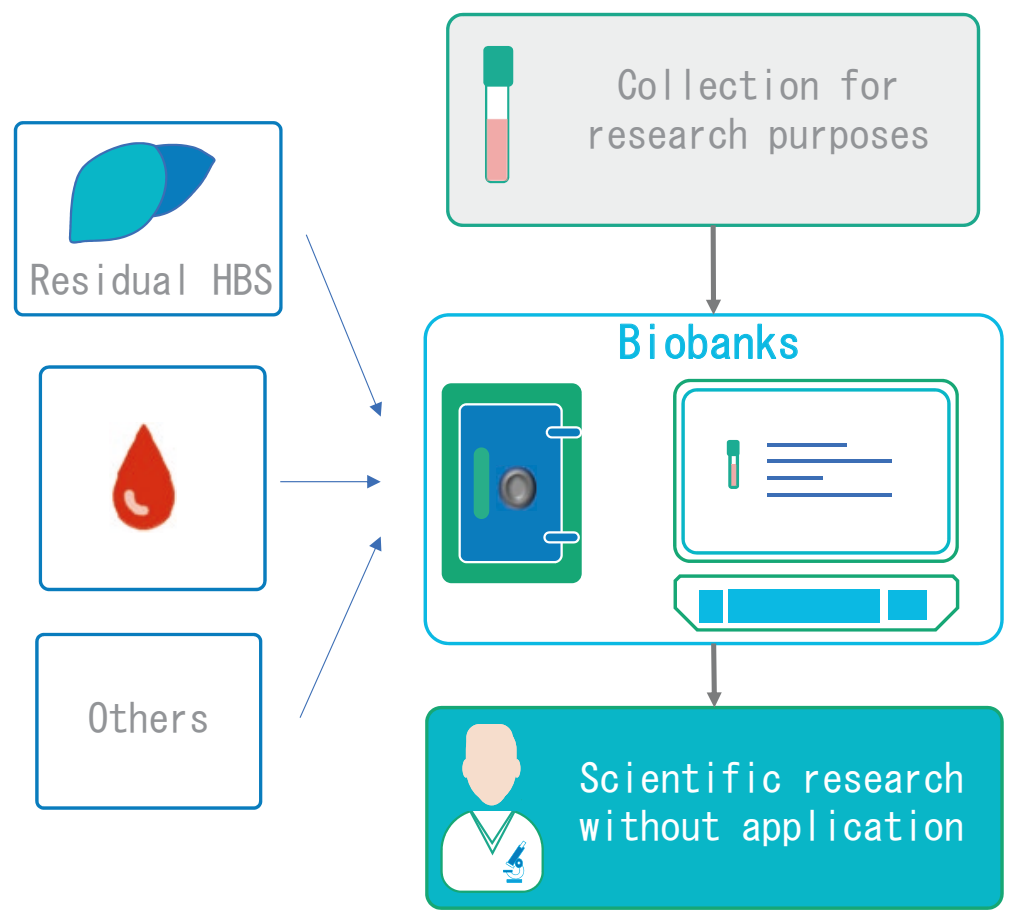

Fig. 1 Concept of biobank: collection of human body material and residual human biological specimen (HBS) 


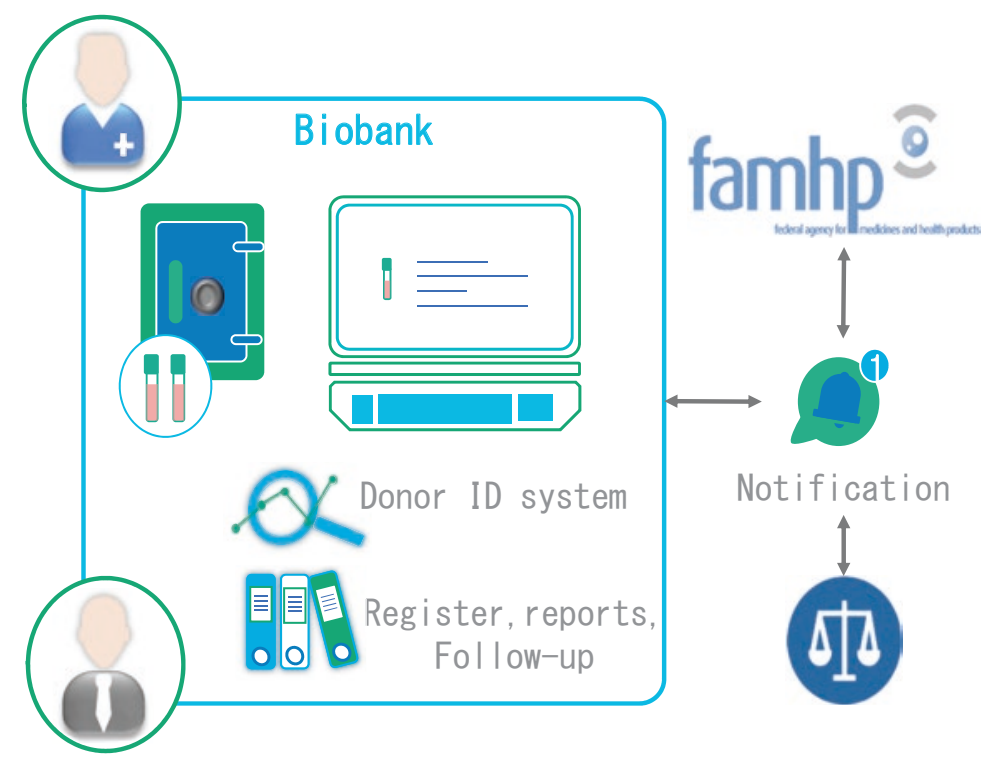

Fig. 2 Notification obligation

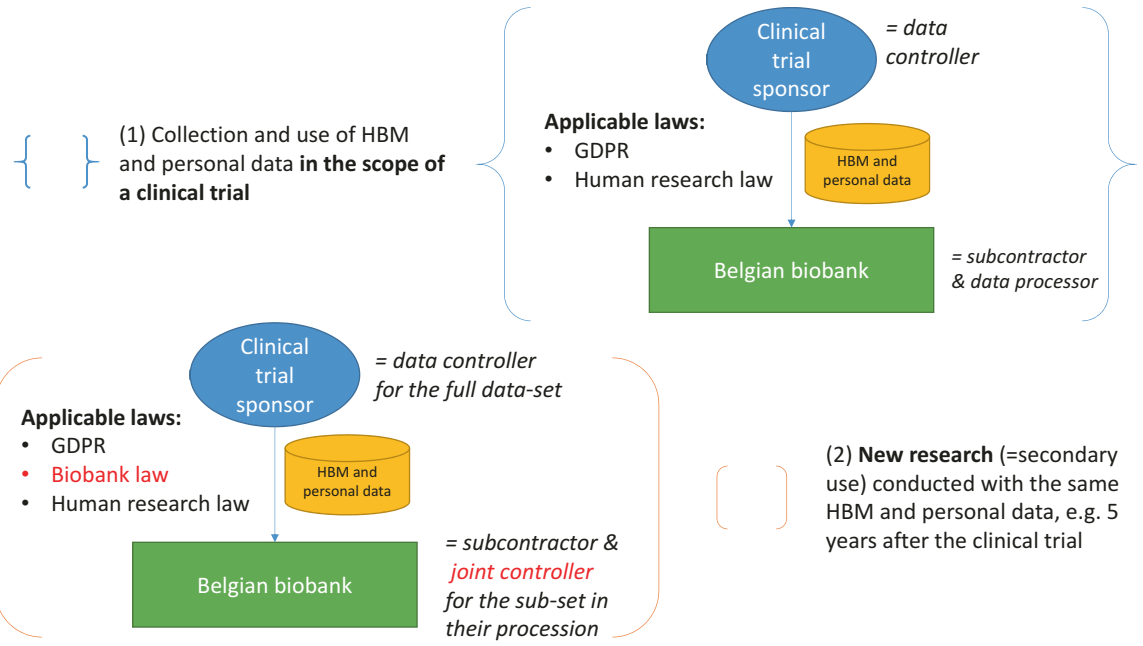

Fig. 3 Secondary use of HBM and personal data outside the scope of a clinical trial 


\section{References}

Allen J, McNamara B (2011) Reconsidering the value of consent in biobank research. Bioethics 25(3):155-166

Belgian Advisory Committee on Bioethics. Opinion No 45 of 19 January 2009

Belgian Privacy Commission. Opinion No 10/2009

De Clercq E, Kaye J, Wolf SM, Koenig B, Bernice E (2017) Returning results in biobank research: global trends and solutions. Genet Test Mol Biomark 21(3):128-131

European Data Protection Board. Opinion 3/2019 concerning the Questions and Answers on the interplay between the Clinical Trials Regulation (CTR) and the General Data Protection regulation (GDPR)

Federal Agency for Medicines and Health Products (2018) Compendium Biobanken. Available at https://www.bbmri.be/wp-content/uploads/2018/08/compendium_20072018_0.pdf

Gottweis H et al (2012) Biobanks for Europe: a challenge for governance. Report of the Expert Group on Dealing with Ethical and Regulatory Challenges of International Biobank Research. Directorate-General for Research and Innovation, European Commission. Brussels. https:// www.coe.int/t/dg3/healthbioethic/Activities/10_Biobanks/biobanks_for_Europe.pdf. Accessed 25 Mar 2019

Kosta E (2011) Untravelling consent in European data protection legislation: a prospective study on consent in electronic communications. PhD Dissertation, KU Leuven

Kozlakidis Z, Cason R, Mant C, Cason J (2012) Human tissue biobanks: the balance between consent and the common good. Res Ethics 8(2):113-123

Morente MM, Cereceda L, Luna-Crespo F et al (2011) Managing a biobank network. Biopreserv Biobank 9(2):187-190

Panis S, Van Gelder N (2008) De Wet Menselijk Lichaamsmateriaal van 19 December 2008: eengrondige analyse. Tijdschr voor Gezondheidsr (4):217-253

Shickle D, Griffin M, El-Arifi K (2010) Inter- and intra-biobank networks: classification of biobanks. Pathobiology. Shickle, D, Academic Unit of Public Health, Institute of Health Sciences, University of Leeds, Leeds, 77(4):181-190

Sterckx S, Van Assche K (2011) The new Belgian law on biobanks: some comments from an ethical perspective. Health Care Anal 19(3):247-258

Verlinden M (2015) Legal framework applicable to access to biobanks. PhD Dissertation, KU Leuven

Open Access This chapter is licensed under the terms of the Creative Commons Attribution 4.0 International License (http://creativecommons.org/licenses/by/4.0/), which permits use, sharing, adaptation, distribution and reproduction in any medium or format, as long as you give appropriate credit to the original author(s) and the source, provide a link to the Creative Commons license and indicate if changes were made.

The images or other third party material in this chapter are included in the chapter's Creative Commons license, unless indicated otherwise in a credit line to the material. If material is not included in the chapter's Creative Commons license and your intended use is not permitted by statutory regulation or exceeds the permitted use, you will need to obtain permission directly from the copyright holder.

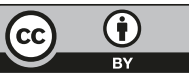

